\title{
P120 catenin expression and its correlation with E-cadherin in salivary gland neoplasms
}

\author{
Ekarat Phattarataratip ${ }^{\mathrm{a}, *}$, Nicha Kositkittiwanit ${ }^{\mathrm{b}}$, Pruch Kajornkiatkul ${ }^{\mathrm{b}}$, Pataraporn Yeunyong ${ }^{\mathrm{b}}$, \\ Ratanatip Ratanapitak ${ }^{\mathrm{b}}$
}

${ }^{a}$ Department of Oral Pathology, Faculty of Dentistry, Chulalongkorn University, Henri-Dunant Road, Pathumwan, Bangkok, 10330, Thailand

${ }^{\mathrm{b}}$ Faculty of Dentistry, Chulalongkorn University, Henri-Dunant Road, Pathumwan, Bangkok, 10330, Thailand

\section{A R T I C L E I N F O}

\section{Keywords:}

P120 catenin

E-cadherin

Mucoepidermoid carcinoma

Adenoid cystic carcinoma

Pleomorphic adenoma

Polymorphous adenocarcinoma

\begin{abstract}
A B S T R A C T
Objectives: Altered P120 catenin expression has been associated with E-cadherin loss and poor prognosis in several cancers. The objectives of this study were to examine the P120 catenin expression in salivary gland neoplasms in correlation with E-cadherin and assess the relationships between their expression levels and pathologic characteristics.

Methods: Fifty-two cases of salivary gland neoplasms, i.e. 25 mucoepidermoid carcinomas (MEC), 13 adenoid cystic carcinomas (ACC), 12 pleomorphic adenomas (PA) and 2 polymorphous adenocarcinomas (PAC) were included. The expression of P120 catenin and E-cadherin was investigated immunohistochemically.

Results: Both P120 catenin and E-cadherin were overexpressed in salivary gland neoplasms, compared to normal tissue. P120 catenin was primarily detected on the membrane of neoplastic cells in most cases. A significant correlation between levels of expression of both proteins was noted in MECs. In ACCs and PA, ductal cells showed positive immunoreactivity, whereas myoepithelial cells variably expressed both proteins. Increased P120 catenin expression was significantly associated with the solid subtype of ACCs.

Conclusions: The cadherin-catenin complex is preserved in the heterogenous tumor cell population in salivary gland neoplasms. Overexpression of P120 catenin may be involved in the progression to solid ACCs.
\end{abstract}

\section{Introduction}

Epithelial tissues are characterized by their ability to form cell-tocell adhesion via several types of junctional units. These structures serve as both physical barriers and regulators of substance transport. ${ }^{1}$ This process utilizes a myriad of adhesion molecules and is fundamental to the morphogenesis, differentiation, function as well as the integrity of tissues. The altered expression of several epithelial adhesion molecules has been shown to play a role in the transformation and progression into cancer cells. ${ }^{2}$

Cadherin and catenin family proteins are vital to the integrity of epithelial tissues through the formation of adherens junctions on the basolateral membrane. E-cadherin is the founding member and key component of cadherin family. ${ }^{3}$ It is extensively studied and well-recognized as a crucial molecule involved in the cancer suppression. The dysfunction or loss of E-cadherin leads to the enhanced epithelial cell migration and invasion. This is crucial in the process of epithelial-mesenchymal transition commonly found in epithelial cancers. ${ }^{4-7}$ Several studies reported the association between the depleted E-cadherin expression and the development or advanced progression in many cancers, including breast, gastric, lung, colon, skin, renal, hepatocellular and esophageal carcinomas. ${ }^{8}$

P120 catenin is one of the armadillo catenins, specifically interacting with the highly conserved cytoplasmic domain of E-cadherin. It acts to stabilize the adherens junctional complex. ${ }^{9}$ In addition, it also serves other cellular processes, such as the cytoskeletal arrangement, cellular signaling and transcription. ${ }^{10}$ The role of P120 catenin in cancer development and progression has been established in several studies. Its function can be varied depending on cancer types. The reduced P120 catenin expression was associated with poor prognosis in patients with pancreatic, colorectal, head and neck and esophageal cancers. ${ }^{11}$ In contrast, it was shown in breast cancers that P120 overexpression induced cancer cell invasion and metastasis. ${ }^{12,13}$

In addition, several studies demonstrated that the altered localization of P120 catenin from the plasma membrane to cytoplasm was associated with the E-cadherin inactivation in breast, bladder, lung,

\footnotetext{
* Corresponding author.

E-mail addresses: Ekarat.P@chula.ac.th (E. Phattarataratip), nicha-mint@hotmail.com (N. Kositkittiwanit), butterfly_bomb@hotmail.com (P. Kajornkiatkul), namm2537@gmail.com (P. Yeunyong), Mie_winnie@hotmail.com (R. Ratanapitak).
} 
Table 1

Characteristics of 52 patients with neoplasms of salivary gland.

\begin{tabular}{lllll}
\hline Characteristics & MEC (25) & ACC (13) & PA (12) & PAC (2) \\
\hline Sex & & & & \\
$\quad$ Male & 10 & 3 & 3 & 1 \\
$\quad$ Female & 15 & 10 & 9 & 1 \\
$\quad$ Male:Female ratio & $1: 1.5$ & $1: 3.3$ & $1: 3$ & $1: 1$ \\
\hline Age (years) & & & & \\
Mean \pm SD & $43 \pm 18.9$ & $39.5 \pm 14.7$ & $36.8 \pm 11.4$ & 60.5 \\
Range & $16-81$ & $16-65$ & $21-62$ & $56-65$ \\
\hline Sites & & & & \\
$\quad$ Palate & 18 & 9 & 8 & 1 \\
Alveolar mucosa & 4 & 1 & 0 & 0 \\
$\quad$ Buccal mucosa & 0 & 1 & 1 & 0 \\
$\quad$ Retromolar mucosa & 2 & 0 & 0 & 0 \\
Floor of mouth & 1 & 2 & 1 & 0 \\
$\quad$ Upper lip & 0 & 0 & 2 & \\
& & & &
\end{tabular}

pancreatic, prostate and gastric cancers. ${ }^{11}$ In E-cadherin-negative breast and colon cancers, the cytoplasmic localization of P120 catenin is associated with the aggressive cancer phenotypes and poor patient survival. $^{14,15}$

E-cadherin has been shown to be differentially expressed in various salivary gland neoplasms. ${ }^{16-18}$ The expression of P120 catenin in salivary gland neoplasms has not been previously reported in the literature. A study in normal mouse salivary gland showed that conditional inactivation of P120 catenin resulted in the abrogation of acinar development, reduction of E-cadherin and transformation to high-grade intraepithelial neoplasia. ${ }^{19}$ This suggested that the altered expression or localization of P120 catenin may potentially take part in salivary gland tumorigenesis.

Therefore, the objectives of this study were to examine the expression of P120 catenin in 4 types of salivary gland neoplasms in correlation with the E-cadherin expression, and to determine the relationship between the levels of expression of both proteins with the pathologic characteristics of these neoplasms.

\section{Materials and methods}

\subsection{Tissue samples}

Paraffin-embedded tissues of 4 types of salivary gland neoplasms, i.e. mucoepidermoid carcinoma (MEC), adenoid cystic carcinoma (ACC), pleomorphic adenoma (PA), and polymorphous adenocarcinoma (PAC), were obtained from the collection of Department of Oral Pathology from 1997 to 2017. Cases with adequate demographic/ clinical data and sufficient tissue specimens were included.

All tissues were reassessed histopathologically. Prognostically relevant microscopic attributes of MECs were documented. These features include the intracystic components $<25 \%$, perineural, vascular or bone invasion, tumor necrosis, increased mitotic activity $>4 / 10$ HPFs, nuclear atypia and tumor front invasion in small nests. MEC was graded based on the histopathologic criteria from Brandwein et al. ${ }^{20}$ ACCs were separated into the cribriform, tubular or solid subtypes, depending on the principal microscopic pattern. This research protocol was authorized by the Human Research Ethics Committee of the Faculty of Dentistry, Chulalongkorn University (HREC-DCU 2017-024).

\subsection{Immunohistochemical methods}

The Bond Polymer Refine Detection kit (Leica Biosystems, Bannockburn, IL) was used for all immunohistochemical staining with Leica Bond-Max Autostainer. Five $\mu \mathrm{m}$-thick sections were deparaffinized with the Bond Dewax Solution and incubating with the Bond Epitope Retrieval Solution 2 for $30 \mathrm{~min}$ at $100^{\circ} \mathrm{C}$. Slides were then applied with $3 \%$ hydrogen peroxide for $5 \mathrm{~min}$. This was followed by $40-$ min incubation with either the anti-P120 catenin antibody (Dilution 1:200, clone MRQ-5, Cell Marque, Rocklin, CA, USA) or anti-E-cadherin antibody (Dilution 1:600, clone EP700Y, Cell Marque, Rocklin, CA, USA) at room temperature. The 8-min incubation each with Post Primary Polymer and Polymer Poly-HRP IgG were then performed, respectively. The Bond Wash Solution was used to rinse slides between steps. Afterwards, diaminobenzidine was applied for $4 \mathrm{~min}$. Slides were then rinsed deionized water and counterstained with hematoxylin. Breast cancer tissues were utilized as positive controls for both P120 catenin and E-cadherin expression. The isotype-matched mouse IgG1 was used in place of both antibodies for negative controls.

\subsection{Immunohistochemical evaluation}

Authors were blinded from the subject clinical or pathological data during the examination of immunohistochemical slides. The percentage of positive tumor cells was semi-quantitatively evaluated and classified into $1-4$ staining levels, i.e. $0=$ no positive cells; $1+=$ percent tumor positivity $<10 \% ; 2+=$ percent tumor positivity between 10 and $50 \%$; $3+=$ percent tumor positivity between 50 and $80 \%$ and $4+=$ percent tumor positivity $>80 \%$. The expression of both P120 catenin and Ecadherin was later divided into low expression (groups 0 and 1) and high expression (groups 2, 3 and 4).

Statistical analyses were performed using the IBM SPSS Statistics version 21 (IBM Corporation, NY) for Windows. The means \pm standard deviation (SD) was used to present the continuous variables. The Pearson's chi-square test or the Fisher's exact test was used to evaluate the correlation between the clinical-pathologic parameters and the P120 catenin or E-cadherin expression. Statistical significance was recognized when $P$-value was less than 0.05 .

Table 2

P120 catenin and E-cadherin expression in neoplasms of salivary gland and normal salivary gland tissues.

\begin{tabular}{|c|c|c|c|c|c|c|c|c|}
\hline \multirow[t]{2}{*}{ Tissues (n) } & \multirow[t]{2}{*}{ Proteins } & \multicolumn{5}{|c|}{ Levels of immunoreactivity, n (\%) } & \multicolumn{2}{|c|}{ Spearman's rho } \\
\hline & & 0 & $1+$ & $2+$ & $3+$ & $4+$ & $\mathrm{r}$ & $P$ value \\
\hline \multirow[t]{2}{*}{ SG (17) } & P120 catenin & $0(0)$ & $16(94.1)$ & $1(5.9)$ & $0(0)$ & $0(0)$ & \multirow[t]{2}{*}{0.685} & \multirow[t]{2}{*}{0.002} \\
\hline & E-cadherin & $0(0)$ & $15(88.2)$ & $2(11.8)$ & $0(0)$ & $0(0)$ & & \\
\hline \multirow[t]{2}{*}{ MEC (25) } & P120 catenin & $8(32.0)$ & $5(20.0)$ & $9(36.0)$ & $1(4.0)$ & $2(8.0)$ & \multirow[t]{2}{*}{0.594} & \multirow[t]{2}{*}{0.002} \\
\hline & E-cadherin & $1(4.0)$ & $4(16.0)$ & $9(36.0)$ & $4(16.0)$ & $7(28.0)$ & & \\
\hline \multirow[t]{2}{*}{ ACC (13) } & P120 catenin & $3(23.1)$ & $7(53.8)$ & $0(0)$ & $3(23.1)$ & $0(0)$ & \multirow[t]{2}{*}{0.534} & \multirow[t]{2}{*}{0.06} \\
\hline & E-cadherin & $0(0)$ & $2(15.4)$ & $6(46.2)$ & $4(30.8)$ & $1(7.7)$ & & \\
\hline \multirow[t]{2}{*}{ PA (12) } & P120 catenin & $0(0)$ & $9(75.0)$ & $2(16.7)$ & $1(8.3)$ & $0(0)$ & \multirow[t]{2}{*}{0.465} & \multirow[t]{2}{*}{0.127} \\
\hline & E-cadherin & $0(0)$ & $0(0)$ & $6(50.0)$ & $3(25.0)$ & $3(25.0)$ & & \\
\hline \multirow[t]{2}{*}{ PAC (2) } & P120 catenin & $0(0)$ & $1(50.0)$ & $0(0)$ & $1(50.0)$ & $0(0)$ & \multirow[t]{2}{*}{1.0} & \multirow[t]{2}{*}{ NA } \\
\hline & E-cadherin & $0(0)$ & $0(0)$ & $1(50.0)$ & $1(50.0)$ & $0(0)$ & & \\
\hline
\end{tabular}


A.

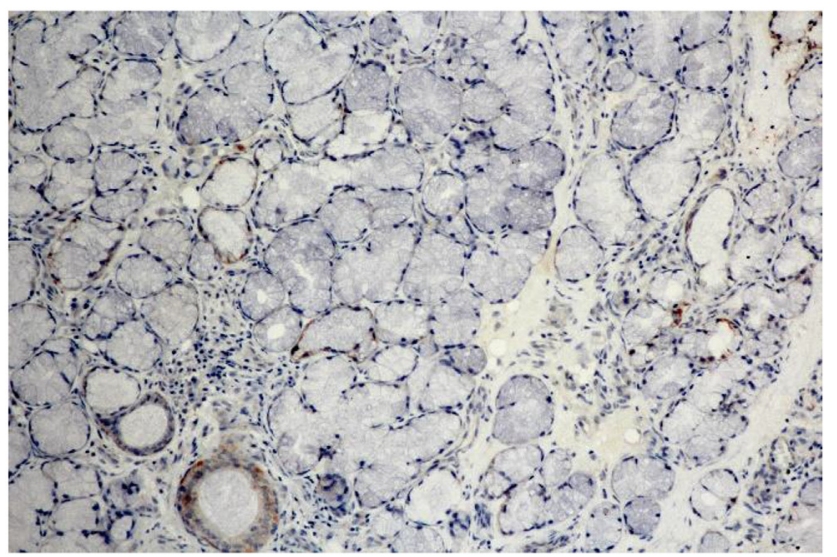

B

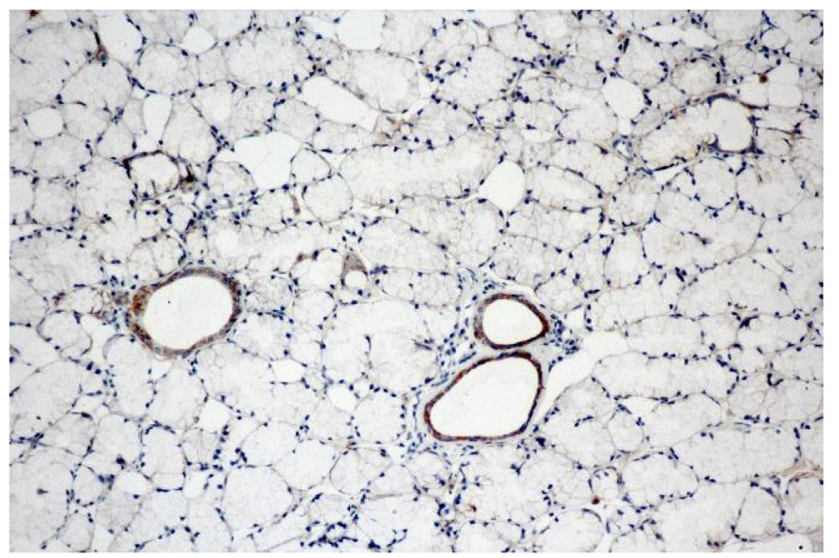

Fig. 1. P120 catenin (A) and E-cadherin (B) expression in normal salivary gland tissue. Both proteins were primarily detected on ductal cells and some myoepithelial cells.

\section{Results}

\subsection{Clinical and demographic data of subjects}

Overall, 52 patients with neoplasms of salivary gland were included. There were 25 MECs, 13 ACCs, 12 PAs and 2 PACs. The average age of subjects was $43 \pm 18.9$ years for MECs, $39.5 \pm 14.7$ years for ACCs, $36.8 \pm 11.4$ years for PAs and 60.5 years for PACs. The ratios of maleto-female were 1:1.5, 1:3.3, 1:3 and 1:1 for MECs, ACCs, PAs and PACs, respectively. Most lesions occurred on the palate (Table 1 ).

\subsection{P120 catenin and E-cadherin expression in neoplasms of salivary gland}

Levels of P120 catenin and E-cadherin expression were shown in Table 2. Four types of salivary gland neoplasms examined showed apparent but variable expression of both P120 catenin and E-cadherin. In MEC, the immunoreactivity was noted primarily on the plasma membrane and less frequently in the cytoplasm of the neoplastic cells for both proteins. In ACCs, PAs and PACs, their expression was limited to the cell membrane.

Normal salivary gland tissue showed minimal P120 catenin and Ecadherin expression. Most specimens stained P120 catenin (94.1\%) and E-cadherin (88.2\%) in less than $10 \%$ of the cells. Their expression was primarily localized to the glandular epithelial cells of salivary ducts and some myoepithelial cells. No expression of both proteins was detected in the salivary acinar cells (Fig. 1).
A.

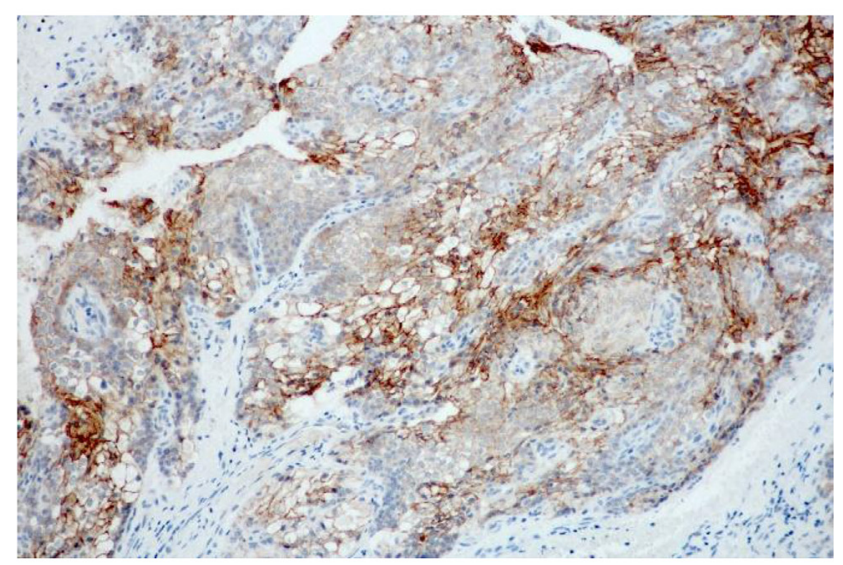

B

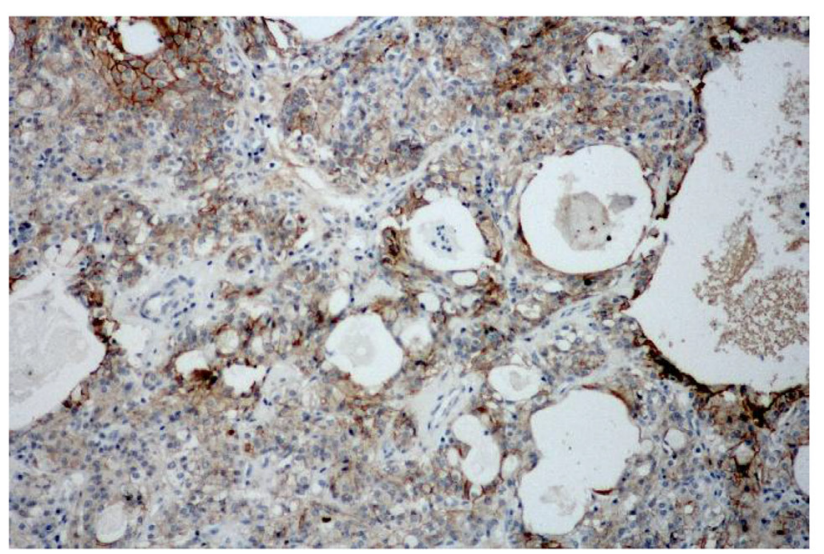

Fig. 2. P120 catenin (A) and E-cadherin (B) expression in MECs. Strong immunoreactivity of both proteins was detected regardless of tumor grades.

In salivary gland neoplasms, the expression of E-cadherin appeared to be greater than that of P120 catenin in most cases. MECs showed markedly diverse expression of P120 catenin and E-cadherin, varying from negative immunoreactivity to positive tumor staining in more than $80 \%$. ACCs demonstrated moderately diverse expression of P120 catenin and E-cadherin. In contrast, the staining pattern of PAs was relatively uniform. The levels of P120 catenin and E-cadherin expression were significantly correlated in both MECs $(\mathrm{P}=0.002)$ and normal salivary gland tissue $(\mathrm{P}=0.002)$.

\subsubsection{Expression in $M E C$}

P120 catenin expression was noted in 68\% of MECs. Most MECs (36\%) expressed P120 catenin between 10 and $50 \%$ of the tumor cells (level $2+$ ). This was followed by no immunoreactivity (level $0,32 \%$ of the cases) and between 0 and $10 \%$ of tumor cells (level $1+$ ), $20 \%$ of the cases, respectively. The pattern of staining was varied among cases. The positive staining was noted primarily on the membrane of squamous cells, non-specific ductal cells and some intermediate cells. Mucoussecreting cells and clear cells were primarily negative (Fig. 2A). Only one case (4\%) showed P120 catenin expression in both cell membrane and cytoplasm.

All but one MEC cases expressed E-cadherin. Most cases (36\%) expressed E-cadherin between 10 and $50 \%$ of the tumor cells (level 2+), followed by more than $80 \%$ of the tumor cells (level $4+, 28 \%$ of the cases). Similar to that of P120 catenin, E-cadherin expression was primarily detected on the membrane of epidermoid cells, intermediate cells and non-specific ductal cells. In addition, the membrane of some 
clear cells was also immunoreactive (Fig. 2B). Seven cases (28\%) showed E-cadherin staining in both cell membrane and cytoplasm of the tumor cells.

Follow-up data were available in 13 MEC cases (52\%) with followup period ranging from 1 to 15 years. No recurrence was observed. The associations between P120 catenin or E-cadherin expression and various pathologic features of MEC were examined. No statistically significant association was observed between the levels or the localization of expression of both proteins and pathologic characteristics of MECs (Table 3).

\subsubsection{Expression in ACC}

Most ACCs (53.8\%) showed expression of P120 catenin in between 0 and $10 \%$ of the tumor cells (level $1+$ ), equally followed by between 50 and $80 \%$ of the tumor cells (level $3+$ ) and no immunoreactivity (level 0 ), constituting $23.1 \%$ of the cases in each group. E-cadherin expression was detected in between 10 and $50 \%$ of the tumor cells (level $2+$ ) in the majority of cases (46.2\%), followed by between 50 and $80 \%$ (level $3+, 30.8 \%$ of the cases) and between 0 and $10 \%$ of tumor cells (level $1+, 15.4 \%$ of cases), respectively (Fig. 3).

The immunoreactivity was noted mainly on the cell membrane for both proteins. Regarding the expression of P120 catenin and E-cadherin in different histopathologic patterns of ACCs. For both proteins, only ductal cells showed positive staining in the cribriform and tubular subtypes. However, in the solid histopathologic pattern, both ductal and myoepithelial cell demonstrated strong immunoreactivity. The low P120 catenin expression was significantly associated with ACCs showing cribriform/tubular histopathologic patterns, whereas solidtyped ACCs expressed high P120 catenin $(\mathrm{P}=0.04)$. The correlation between the expression levels of E-cadherin and histopathologic patterns of ACCs was not of statistical significance (Table 3).

A.

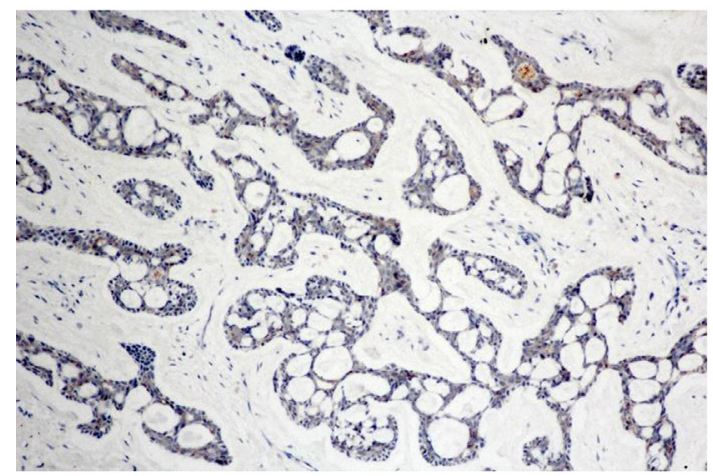

C.

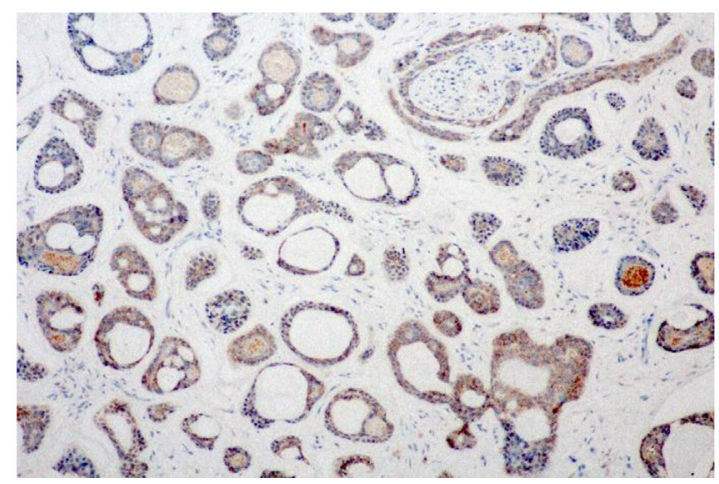

Table 3

Correlation between P120 catenin and E-cadherin expression and pathologic characteristics of MEC and ACC.

\begin{tabular}{|c|c|c|c|c|c|c|}
\hline \multirow[t]{2}{*}{ Pathologic features } & \multicolumn{3}{|c|}{ P120 catenin } & \multicolumn{3}{|c|}{ E-cadherin } \\
\hline & Low & High & $P$-value & Low & High & $P$-value \\
\hline \multicolumn{7}{|l|}{ MEC } \\
\hline \multicolumn{7}{|l|}{ Cystic component < 25\% } \\
\hline Present & 6 & 4 & 0.69 & 2 & 8 & 1.00 \\
\hline Absent & 7 & 8 & & 3 & 12 & \\
\hline \multicolumn{7}{|c|}{ Tumor fronts invading in small nests } \\
\hline Present & 6 & 8 & 0.43 & 1 & 13 & 0.133 \\
\hline Absent & 7 & 4 & & 4 & 7 & \\
\hline \multicolumn{7}{|l|}{ Anaplasia } \\
\hline Present & 3 & 4 & 0.67 & 0 & 7 & 0.27 \\
\hline Absent & 10 & 8 & & 5 & 13 & \\
\hline \multicolumn{7}{|l|}{ Necrosis } \\
\hline Present & 0 & 2 & 0.22 & 0 & 2 & 1.00 \\
\hline Absent & 13 & 10 & & 5 & 18 & \\
\hline \multicolumn{7}{|l|}{ Mitosis > 4/HPFs } \\
\hline Present & 0 & 1 & 0.48 & 0 & 1 & 1.00 \\
\hline Absent & 13 & 11 & & 5 & 19 & \\
\hline \multicolumn{7}{|l|}{ Bone invasion } \\
\hline Present & 0 & 2 & 0.22 & 0 & 2 & 1.00 \\
\hline Absent & 13 & 10 & & 6 & 18 & \\
\hline \multicolumn{7}{|l|}{ Vascular invasion } \\
\hline Present & 0 & 2 & 0.22 & 0 & 2 & 1.00 \\
\hline Absent & 13 & 10 & & 5 & 18 & \\
\hline \multicolumn{7}{|l|}{ Pathologic grade } \\
\hline Low/Intermediate grade & 9 & 7 & 0.69 & 5 & 11 & 0.12 \\
\hline High grade & 4 & 5 & & 0 & 9 & \\
\hline \multicolumn{7}{|l|}{ ACC } \\
\hline \multicolumn{7}{|l|}{ Pathologic subtypes } \\
\hline Cribriform/Tubular & 10 & 1 & 0.04 & 2 & 9 & 1.00 \\
\hline Solid & 0 & 2 & & 0 & 2 & \\
\hline
\end{tabular}

B

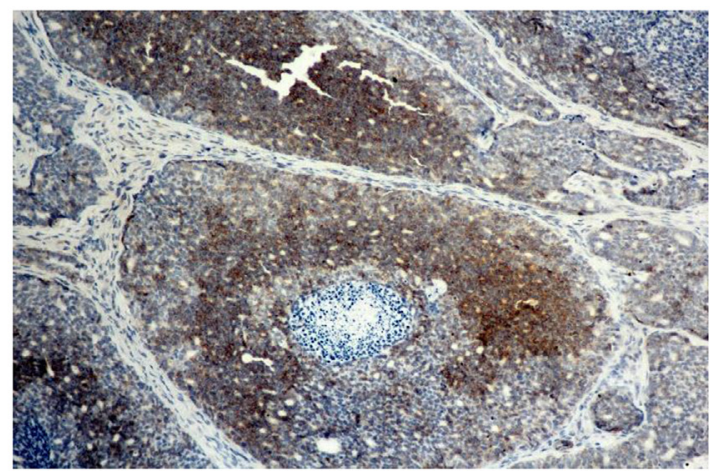

$\mathrm{D}$

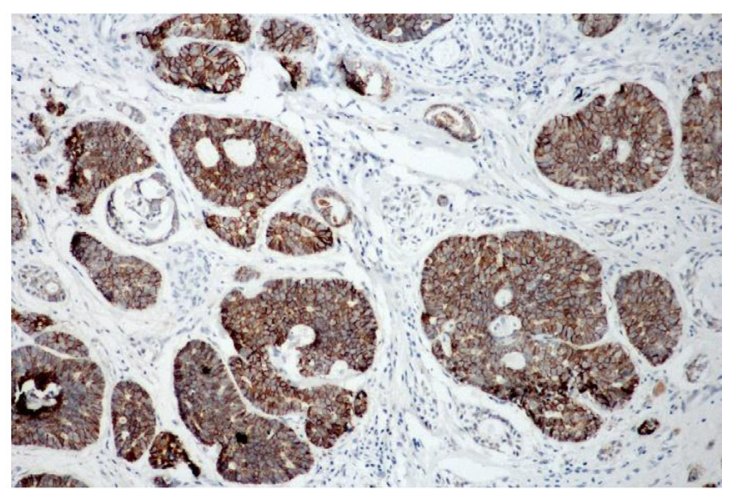

Fig. 3. P120 catenin and E-cadherin expression in ACCs. Mild P120 catenin immunoreactivity was detected primarily on ductal cells of the cribriform/tubular subtype of ACCs(A), whereas solid subtype (B) showed strong expression. E-cadherin showed strong expression of both cribriform/tubular (C) and solid (D) subtypes. 
A.

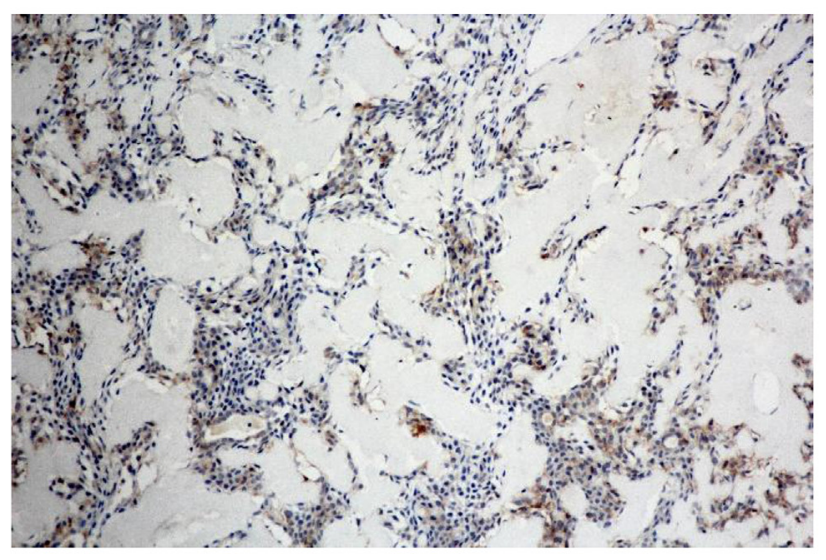

B

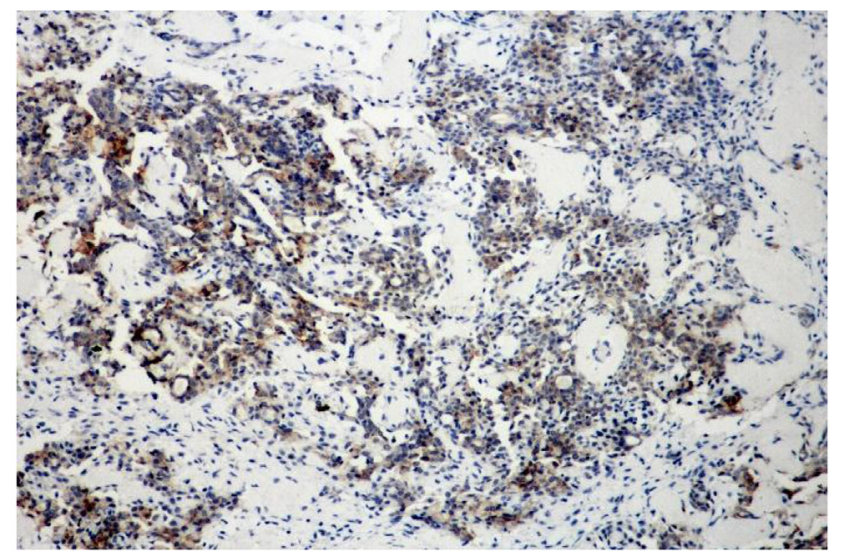

Fig. 4. P120 catenin (A) and E-cadherin (B) expression in PAs. Ductal cells uniformly showed positive immunoreactivity to both proteins, whereas the staining was varied in myoepithelial cells among cases.

\subsubsection{Expression in $P A$}

All PAs expressed both P120 catenin and E-cadherin. Most cases (75.0\%) showed P120 catenin expression between 0 and $10 \%$ of the tumor cells (level $1+$ ), followed by between 10 and $50 \%$ of the tumor cells (level 2+, 16.7\% of cases) (Fig. 4A). Half of the PA cases expressed E-cadherin between 10 and 50\% of the tumor cells (level $2+$ ). The remaining cases showed expression between 50 and $80 \%$ (level $3+, 25 \%$ of the cases) and more than $80 \%$ of the tumor cells (level $4+$, $25 \%$ of cases). The expression of both proteins was noted mainly on the membrane of ductal cells and some myoepithelial cells (Fig. 4B).

\subsubsection{Expression in PAC}

Two PAC cases expressed both P120 catenin and E-cadherin on the cell membrane. One case showed expression of both proteins between 50 and $80 \%$ of tumor cells. The other case expressed P120 catenin between 0 and $10 \%$ of tumor cells and E-cadherin between 10 and $50 \%$ of the tumor cells (Fig. 5).

\section{Discussion}

In this study, we reported the differential expression of P120 catenin in correlation with E-cadherin in 4 distinct types of salivary gland neoplasms. The expression of both proteins was increased in neoplasms compared to normal salivary tissue, with P120 catenin and E-cadherin being expressed in $78.9 \%$ and $98.1 \%$ of the cases, respectively. The
A.

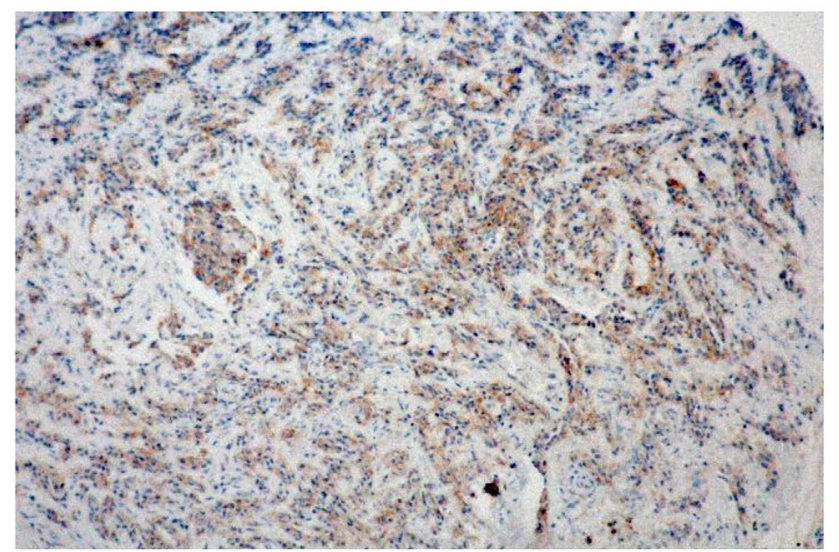

B

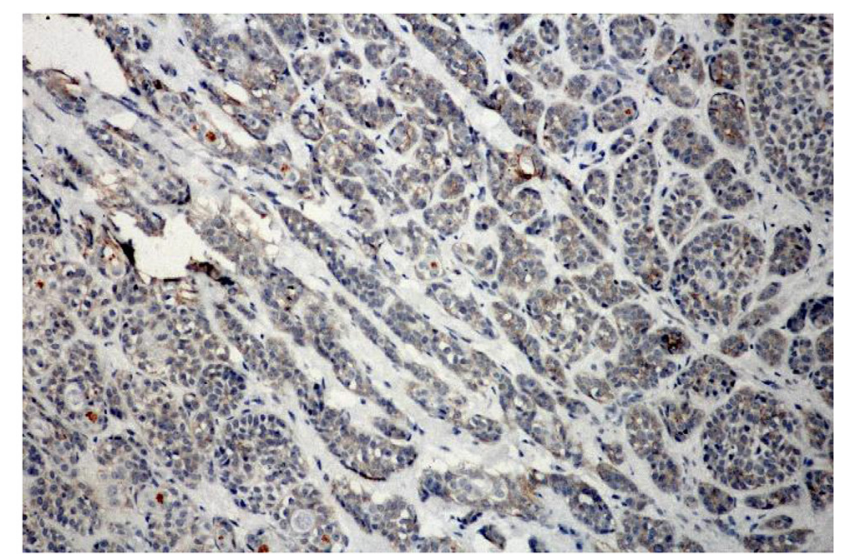

Fig. 5. P120 catenin (A) and E-cadherin (B) expression in PACs. Neoplastic cells in PACs showed positive immunoreactivity to both proteins.

level of P120 catenin expression was generally lower compared to that of E-cadherin. In normal salivary gland tissue, P120 catenin and Ecadherin expression was limited and primarily localized in the membrane of ductal cells and a few myoepithelial cells. Correspondingly in neoplasms, the glandular epithelial cells in MECs, PAs and ACCs remained largely positive for both proteins. Other neoplastic cell populations demonstrated variation in staining pattern. The disparity in expression among neoplasms partly depends on the different amount of each distinct type of tumor cells present in individual cases.

In MECs, the squamous cells and intermediate cells showed variation in number and intensity of positive staining among cases, whereas the mucous-secreting cells and clear cells were primarily negative. Our data supported the findings from previous studies that the strong Ecadherin expression was noted in neoplasms of salivary glands. ${ }^{16,18,21}$ Its level of expression was not correlated with the prognostically related pathologic characteristics of MECs. ${ }^{17}$ In addition, we did not find correlations between the pathologic characteristics of MECs and the P120 catenin expression. Significantly, we found the positive correlation between the levels of P120 catenin and E-cadherin in MECs, suggesting that both proteins tend to co-localize in this neoplasm.

Regarding PAs and ACCs, P120 catenin expression was present in most cases albeit at a relatively low level. This could be that the staining was primarily limited to the neoplastic ductal cells, which represented a smaller amount in the mixed population of neoplastic cells in both neoplasms. The differential expression was in part depended on the 
number of ductal cells present and the variation in immunoreactivity of myoepithelial cells among cases. The expression of E-cadherin was present in all PA, ACC and PAC cases. We substantiated the data from Cavalcante et al., which observed the decreased expression of E-cadherin in ACCs, compared to PAs. ${ }^{22}$ It would be of interest to further elucidate whether the loss of E-cadherin is associated with the malignant potential of ACCs.

The loss of E-cadherin was shown to be associated with the cytoplasmic localization of P120 catenin in several cancers. ${ }^{14,15,23,24}$ In this study, we found that in the 4 salivary gland neoplasms examined, the expression of P120 catenin is principally membranous of varying intensity. The cytoplasmic localization of P120 catenin is infrequent and only noted in a portion of neoplastic cells in one case of MEC. Together with the preserved membranous E-cadherin expression, our data suggested that the cadherin-catenin complex is maintained on the neoplastic cell surface in these neoplasms. These results also correspond to the study on genetic level, showing that E-cadherin gene (CDH1)-160C/ A polymorphism is uncommon and not related to the E-cadherin expression in PAs and ACCs. ${ }^{22}$

In ACCs, it is well-characterized that the solid histopathologic pattern is associated with the worse patient prognosis than either the cribriform or tubular subtype. Significantly, we found the higher P120 catenin expression in solid-subtyped ACCs than those with cribriform/ tubular subtype. This finding is of interest in that P120 catenin has been shown to exert the pro-tumorigenic activity in cancers. In breast cancer cell lines, P120 catenin could mediate the ErbB2-induced migration and invasion, via the Rac1 and Cdc42 activation, without the change in Ecadherin expression. In addition, the overexpression of P120 catenin in the ErbB2-positive cancer cell increased its ability to metastasize in vivo. ${ }^{12}$ In inflammatory breast cancers, the P120 catenin/E-cadherin complex overexpression is associated with the increased invasion and the formation of tumor emboli in response to eIF4GI overexpression. ${ }^{13}$ Therefore, it could be valuable to examine in future studies whether the P120 overexpression in solid ACCs is associated with the enhanced invasion and overall aggressiveness of this subtype.

The potential therapeutic interventions for cancers with altered or loss of P120 catenin/E-cadherin expression have been implicated. However, any regimens will likely need to customize to specific tumor type. ${ }^{1}$ Soto et al. suggested that targeting Rac and MAPK signaling might be beneficial in treating E-cadherin-silenced breast cancers from hypermethylation. Alternatively, those with mutant E-cadherin and cytoplasmic localization of P120 catenin might benefit from ROCK inhibitors. ${ }^{25}$ In E-cadherin-deficient renal cell carcinomas, Yanagisawa et al. suggested that the $\mathrm{N}$-terminal domain of P120 catenin could be a therapeutic target. ${ }^{26}$ In addition, a recent study in lung adenocarcinoma showed that the abnormal P120 catenin expression was associated with the increased resistance to EGFR tyrosine kinase inhibitors. This suggested that P120 catenin might also be useful as a predictive marker of therapeutic outcome. ${ }^{27}$

In conclusion, we provide evidence that both P120 catenin and Ecadherin are overexpressed in salivary gland neoplasms. The levels of P120 catenin and E-cadherin expression are significantly correlated in MECs. The differential expression of both proteins partly corresponds to the varying population of neoplastic cells present in salivary gland neoplasms. The cytoplasmic localization of P120 catenin is a rare event in these neoplasms. P120 catenin may play a role in the progression to the more aggressive solid histopathologic subtype of ACC.

\section{Disclosures}

This work was supported by the Dental Research Fund of the Faculty of Dentistry, Chulalongkorn University, Bangkok, Thailand. All authors disclose no commercial associations or financial interest that might pose a conflict of interest.

\section{Acknowledgement}

This study was funded by the Dental Research Grant of the Faculty of Dentistry, Chulalongkorn University, Bangkok, Thailand.

\section{Appendix A. Supplementary data}

Supplementary data to this article can be found online at https:// doi.org/10.1016/j.jobcr.2018.09.005.

\section{References}

1. Schackmann RC, Tenhagen M, van de Ven RA, Derksen PW. p120-catenin in cancer mechanisms, models and opportunities for intervention. $J$ Cell Sci. 2013;126:3515-3525.

2. Anastasiadis PZ, Reynolds AB. The p120 catenin family: complex roles in adhesion, signaling and cancer. J Cell Sci. 2000;113(Pt 8):1319-1334.

3. Gul IS, Hulpiau P, Saeys Y, van Roy F. Evolution and diversity of cadherins and catenins. Exp Cell Res. 2017;358:3-9.

4. Paredes J, Figueiredo J, Albergaria A, et al. Epithelial E- and P-cadherins: role and clinical significance in cancer. Biochim Biophys Acta. 2012;1826:297-311.

5. Gheldof A, Berx G. Cadherins and epithelial-to-mesenchymal transition. Prog Mol Biol Trans Sci. 2013;116:317-336.

6. Berx G, van Roy F. Involvement of members of the cadherin superfamily in cancer. Cold Spring Harb Perspect Biol. 2009;1:a003129.

7. Kourtidis A, Lu R, Pence LJ, Anastasiadis PZ. A central role for cadherin signaling in cancer. Exp Cell Res. 2017;358:78-85.

8. Birchmeier W, Behrens J. Cadherin expression in carcinomas: role in the formation of cell junctions and the prevention of invasiveness. Biochim Biophys Acta. 1994;1198:11-26.

9. Reynolds AB, Carnahan RH. Regulation of cadherin stability and turnover by p120ctn: implications in disease and cancer. Semin Cell Dev Biol. 2004;15:657-663.

10. Reynolds AB, Roczniak-Ferguson A. Emerging roles for p120-catenin in cell adhesion and cancer. Oncogene. 2004;23:7947-7956

11. Thoreson MA, Reynolds AB. Altered expression of the catenin p120 in human cancer: implications for tumor progression. Differentiation. 2002;70:583-589.

12. Johnson E, Seachrist DD, DeLeon-Rodriguez CM, et al. HER2/ErbB2-induced breast cancer cell migration and invasion require p120 catenin activation of Rac1 and Cdc42. J Biol Chem. 2010;285:29491-29501.

13. Silvera D, Arju R, Darvishian F, et al. Essential role for eIF4GI overexpression in the pathogenesis of inflammatory breast cancer. Nat Cell Biol. 2009;11:903-908.

14. Bellovin DI, Bates RC, Muzikansky A, Rimm DL, Mercurio AM. Altered localization of p120 catenin during epithelial to mesenchymal transition of colon carcinoma is prognostic for aggressive disease. Canc Res. 2005;65:10938-10945.

15. Shibata T, Kokubu A, Sekine S, Kanai Y, Hirohashi S. Cytoplasmic p120ctn regulates the invasive phenotypes of E-cadherin-deficient breast cancer. Am J Pathol 2004; $164: 2269-2278$.

16. Andreadis D, Epivatianos A, Mireas G, et al. Immunohistochemical detection of Ecadherin in certain types of salivary gland tumours. $J$ Laryngol Otol. 2006;120:298-304.

17. Economopoulou P, Hanby A, Odell EW. Expression of E-cadherin, cellular differentiation and polarity in epithelial salivary neoplasms. Oral Oncol. 2000;36:515-518.

18. Furuse C, Cury PR, Altemani A, dos Santos Pinto Jr D, de Araujo NS, de Araujo VC. Beta-catenin and E-cadherin expression in salivary gland tumors. Int J Surg Pathol. 2006;14:212-217.

19. Davis MA, Reynolds AB. Blocked acinar development, E-cadherin reduction, and intraepithelial neoplasia upon ablation of p120-catenin in the mouse salivary gland. Dev Cell. 2006;10:21-31.

20. Brandwein MS, Ivanov K, Wallace DI, et al. Mucoepidermoid carcinoma: a clinicopathologic study of 80 patients with special reference to histological grading. Am J Surg Pathol. 2001;25:835-845.

21. Prabhu S, Kaveri H, Rekha K. Benign, malignant salivary gland tumors: comparison of immunohistochemical expression of e-cadherin. Oral Oncol. 2009;45:594-599.

22. Cavalcante RB, Nonaka CF, Rabenhorst SH, da Costa Miguel MC, Pinto LP, de Souza LB. Pleomorphic adenoma and adenoid cystic carcinoma of salivary glands: E-cadherin immunoexpression and analysis of the CDH1 -160C/A polymorphism. Arch Oral Biol. 2017;73:48-54.

23. Sarrio D, Perez-Mies B, Hardisson D, et al. Cytoplasmic localization of $\mathrm{p} 120 \mathrm{ctn}$ and $\mathrm{E}-$ cadherin loss characterize lobular breast carcinoma from preinvasive to metastatic lesions. Oncogene. 2004;23:3272-3283.

24. Chetty R, Jain D, Serra S. p120 catenin reduction and cytoplasmic relocalization leads to dysregulation of E-cadherin in solid pseudopapillary tumors of the pancreas. Am J Clin Pathol. 2008;130:71-76.

25. Soto E, Yanagisawa M, Marlow LA, Copland JA, Perez EA, Anastasiadis PZ. p120 catenin induces opposing effects on tumor cell growth depending on E-cadherin expression. J Cell Biol. 2008;183:737-749.

26. Yanagisawa M, Huveldt D, Kreinest P, et al. A p120 catenin isoform switch affects Rho activity, induces tumor cell invasion, and predicts metastatic disease. $J$ Biol Chem. 2008;283:18344-18354.

27. Liu Y, Wang S, Dong QZ, et al. The expression pattern of p120-catenin is associated with acquired resistance to epidermal growth factor receptor tyrosine kinase inhibitors in non-small cell lung cancer. Appl Immunohistochem Mol Morphol. 2018;26:64-70. 